\title{
ASPECTOS REGIO- E ESTEREOQUÍMICOS DA ADIÇÃO DE MICHAEL ASSIMÉTRICA VIA IMINAS QUIRAIS COM CENTROS ESTEREOGÊNICOS PRÉ-EXISTENTES
}

Rossana A. Schenato

Faculdade de Química, Pontifícia Universidade Católica do Rio Grande do Sul, Av. Ipiranga, 6681, 90619-900 Porto Alegre - RS Éverton M. dos Santos, Beatriz S. M. Tenius e Eduardo R. de Oliveira*

Instituto de Química, Universidade Federal do Rio Grande do Sul, CP 15003, 91501-970 Porto Alegre - RS

Recebido em 20/8/02; aceito em 21/2/03

REGIO- AND STEREOCHEMICAL ASPECTS OF THE ASYMMETRIC MICHAEL ADDITION USING CHIRAL IMINES BEARING A RESIDENT STEREOGENIC CENTER. The asymmetric Michael addition reactions using chiral imines, under neutral conditions (deracemizing alkylation process), constitute one of the main methods for the stereocontrolled elaboration of quaternary carbon centers. This protocol is based on the conjugate addition of secondary chiral enamines to electron-deficient alkenes. The focus of this report deals with the discussion of regio- and stereochemical aspects of the deracemizing alkylation process concerning enamines bearing a resident chiral center.

Keywords: deracemizing alkylation process; chiral imines; transmission of chirality.

\section{INTRODUÇÃO}

A reação de Michael assimétrica via iminas quirais, também conhecida por alquilação desracemizante, foi descrita em 1985 por d'Angelo e Pfau e tornou-se uma das metodologias mais empregadas na elaboração estereocontrolada de centros carbônicos quaternários ${ }^{1}$. Esta reação está fundamentada na adição conjugada de iminas quirais a alquenos eletronicamente deficientes. Os aspectos sintéticos e mecanísticos desta metodologia estão revisados em excelentes manuscritos ${ }^{2-4}$.

Iminas quirais do tipo 1 obtidas a partir de ciclanonas 2-substituídas racêmicas 2 e da 1 -feniletilamina opticamente ativa (3) reagem, sob condições neutras, com aceptores de Michael $\mathbf{4}$ produzindo adutos, como $\mathbf{5}$, com alto grau de regio- e estereosseletividade. A hidrólise destes adutos conduz a ciclanonas 2,2-dissubstituídas $\mathbf{6}$, com a recuperação do auxiliar quiral $\mathbf{3}$ (sem perda da atividade óptica, podendo ser reaproveitado após simples purificação) (Esquema 1)².

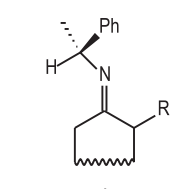

1
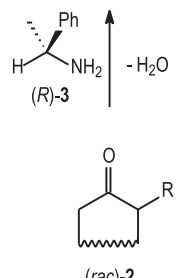

$(\mathrm{rac})-2$

GRE = Grupo Retirador de Elétrons

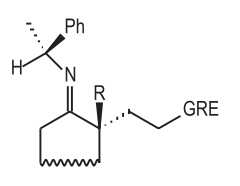

5

$\mathrm{H}_{3} \mathrm{O}^{+}$
Esquema 1. Alquilação desracemizante de uma ciclanona 2-substituída 2 racêmica

*e-mail: eroliv@iq.ufrgs.br
A reação de alquilação desracemizante pode ser estendida a uma grande variedade de ciclanonas ${ }^{5-8}$ e aceptores de Michael (vinilcetonas $^{1}$, anidrido maleico ${ }^{4}$, acrilamidas ${ }^{4}$, acrilatos ${ }^{6}$, acrilonitrilas ${ }^{7,8}$, nitroalquenos ${ }^{9}$, vinilsulfonas ${ }^{10}$, etc.) e tem sido utilizada por nós ${ }^{11} \mathrm{e}$ por vários outros grupos de pesquisa na síntese de produtos naturais, entre os quais estãoterpenos, esteróides, alcalóides, etc. ${ }^{3,10-14}$.

\section{ASPECTOS MECANÍSTICOS}

Os aspectos mecanísticos desta reação de Michael podem ser racionalizados da seguinte maneira: i) a espécie nucleofílica, envolvida neste processo, é a enamina secundária mais substituída que está em equilíbrio tautomérico com a imina $\mathbf{1}$ e ii) fortes evidências indicam um estado de transição cíclico e com a transferência concertada do hidrogênio ligado ao átomo de nitrogênio, da enamina secundária, à posição $\alpha$-GRE do alqueno eletrofílico ${ }^{2-4}$.

Algumas experiências com materiais de partida deuterados confirmam estas considerações ${ }^{15}$. Por exemplo, a adição da imina deuterada 7, derivada da $(R)$-1-feniletiamina (3), ao acrilato de metila (9) produz, após hidrólise, o aduto (2S,2' $S$ )-10, contendo o átomo de deutério na posição $\alpha$-carboxila. $O$ controle absoluto da configuração de C-2' estabelece que o átomo de deutério é transferido do nitrogênio da enamina para o $\mathrm{C}$ - $\alpha$ éster, de forma concertada à formação da ligação C-C e com a aproximação endo entre as espécies reagentes (Figura 1a). Da mesma forma, a adição da imina 11 ao 2deuteroacrilato de etila (13) conduz ao aduto deuterado $\left(2 S, 2^{\prime} R\right)-\mathbf{1 4}$, que difere do aduto $\mathbf{1 0}$ somente na configuração do centro estereogênico C-2' (Esquema 2).

Em geral, esta adição de Michael apresenta alta regiosseletividade, de forma que a alquilação ocorre, predominantemente, na posição $\alpha$ mais substituída da imina. A razão se encontra no equilíbrio tautomérico entre a imina quiral $\mathbf{1}$ e as duas enaminas secundárias 15 e 16 representadas pelos seus confôrmeros mais estáveis, de forma a minimizar a tensão alílica $\mathrm{A}^{1,3}$. Na enamina mais substituída 15, a ligação N-H está sin à ligação dupla da enamina e, conseqüentemente, a transferência interna e concertada do hidrogênio é facilitada. Entretanto, na enamina menos substituída 16 esta transferência concertada não ocorre, devido à geometria da ligação N-H estar anti à ligação dupla desta enamina (Esquema 3$)^{2-4}$. 

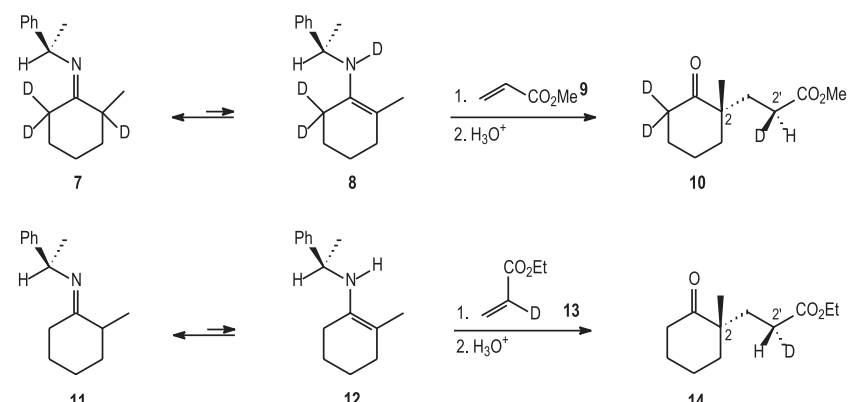

Esquema 2. Alquilação desracemizante empregando os precursores deuterados 7 e 11

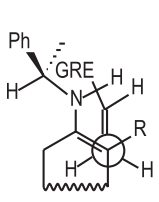

a

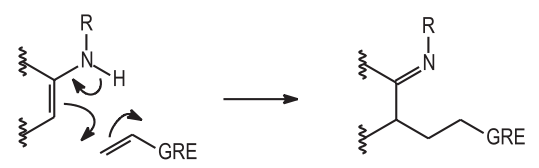

Sintese aza-ene

Figura 1. (a) aproximação endo-sin entre as espécies reagentes e (b) estado de transição cíclico tipo síntese aza-ene
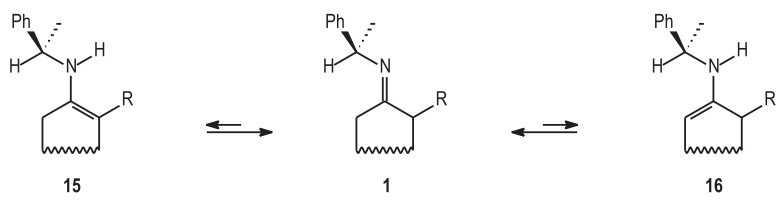

Esquema 3. Equilíbrio tautomérico imina 1 - enaminas 15 e 16

Portanto, a adição de iminas quirais a alquenos eletrofílicos se dá por aproximação endo - sin entre as espécies reagentes (Figura 1a) e, de acordo com d'Angelo, com estado de transição cíclico, característico de uma reação aza-ene, onde há a transferência concertada do próton do nitrogênio da enamina secundária ao aceptor de Michael (Figura 1b) ${ }^{2}$. Ainda, o processo de alquilação ocorre na face $\pi$ menos impedida (anti ao substituinte fenila) ${ }^{2-4}$, no rotâmero mais estável da enamina secundária - proposição baseada em cálculos ab initio realizados por Sevin e colaboradores, empregando as espécies vinilamina e propenal como modelos ${ }^{16}$. Em trabalho posterior $^{17}$, utilizando cálculos semi-empíricos e $a b$ initio em vinilaminas mais complexas, concluiu-se que a piramidalização do átomo de nitrogênio da enamina quiral, comprovada experimentalmente por cristalografia $^{18}$, é um fator que dificulta a adoção de um modelo teórico que explique adequadamente a estereosseletividade observada na reação de alquilação desracemizante. De qualquer forma, a proposição do modelo compacto (Figura 1) é favorecida, considerando a facilidade de transferência interna do hidrogênio da enamina ao aceptor de Michael.

\section{Considerações regioquímicas}

A adição de Michael de iminas a alquenos eletrofílicos normalmente ocorre, com boa regiosseletividade, na posição $\alpha$ mais substituída da imina quiral. Entretanto, há casos onde se observa a perda parcial ou a inversão completa da regioquímica ${ }^{2,9,19}$.

A perda da regioquímica na adição de Michael via iminas quirais tem sido observada em várias situações. A literatura registra que interações estéricas fortes, na enamina secundária mais substituída, podem induzir à perda, ou mesmo à inversão, da regiosseletividade. No entanto, há casos em que mesmo o aceptor de Michael, ou o auxiliar quiral, são suficientes para provocar este resultado regioquímico ${ }^{2}$.

A perda do controle regioquímico foi observada na adição de Michael da imina 18, obtida a partir da tetralona 17 e da $(R)$-PEA (3), à metilvinilcetona (20). A reação conduz à mistura 1:1 dos compostos 21 e $(S)$-22 (88\% e.e.), com rendimento total de $50 \%$ (Esquema 4$)^{20}$. A fenantrona 22 é oriunda da alquilação da enamina $\mathbf{1 9}$, desfavorecida devido à interação alílica $\mathrm{A}^{1,3} \mathrm{e}$ a enona 21 é derivada da alquilação da enamina menos substituída, regioisomérica de $\mathbf{1 9}$.
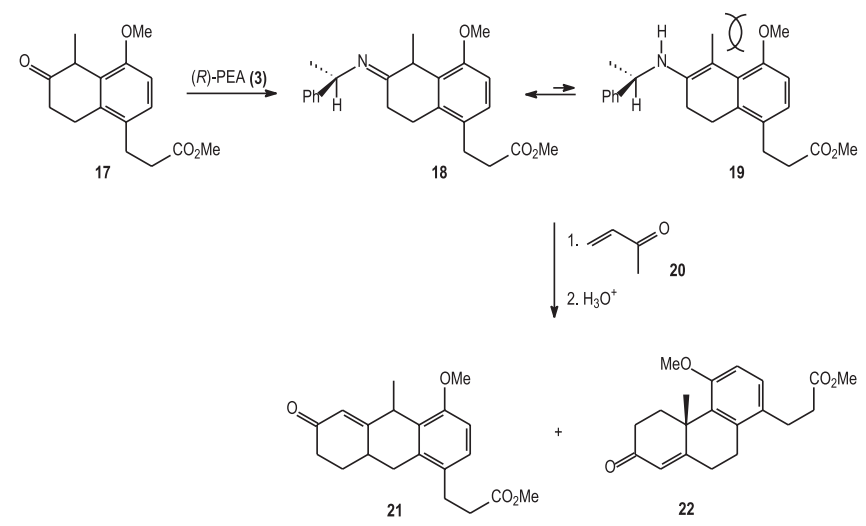

Esquema 4. Adição de Michael da imina 18 à metilvinilcetona (20)

A perda da regiosseletividade, nesta reação, está relacionada a interações estéricas fortes na enamina secundária mais substituída 19, que desestabiliza este tautômero, induzindo a adição via enamina secundária menos substituída. Esta explicação pode ser confirmada através da reação de alquilação desracemizante do análogo desmetoxilado de 17, onde a adição de Michael da sua enamina secundária à metilvinilcetona leva, exclusivamente, ao aduto quaternário ${ }^{20}$.

Entretanto, foi verificado que a adição de Michael da imina 23, derivada da (S)-PEA (3), ao 1,1-bis-fenilsulfonileteno (24) ocorre com inversão total de regiosseletividade. Esta reação produz somente o aduto terciário 25, como mistura de diastereoisômeros (Esquema 5$)^{21}$.

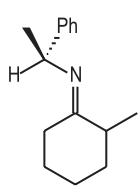

23
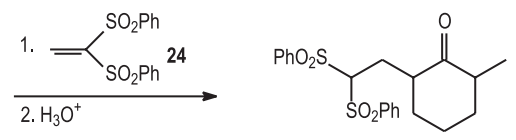

25
Esquema 5. Adição de Michael da imina 23 ao 1,1-bis-fenilsulfonileteno (24)

A adição da imina $(S)$-26, prepararada a partir da 2-metilciclopentanona racêmica e da $(S)$-PEA (3), ao nitroeteno (27) produz a mistura 1:1 dos regioisômeros $\mathbf{2 8}$ e $\mathbf{2 9}$, como mistura de 
diastereoisômeros. Entretanto, a adição de Michael da imina $(S)$-26 ao aceptor 2-nitropropeno (30) é altamente regiosseletiva, produzindo somente os adutos quaternários $\left(2 R, 1^{\prime} S\right)-31$ e $\left(2 R, 1^{\prime} R\right)-32$, na proporção de 9:1 (Esquema 6) .
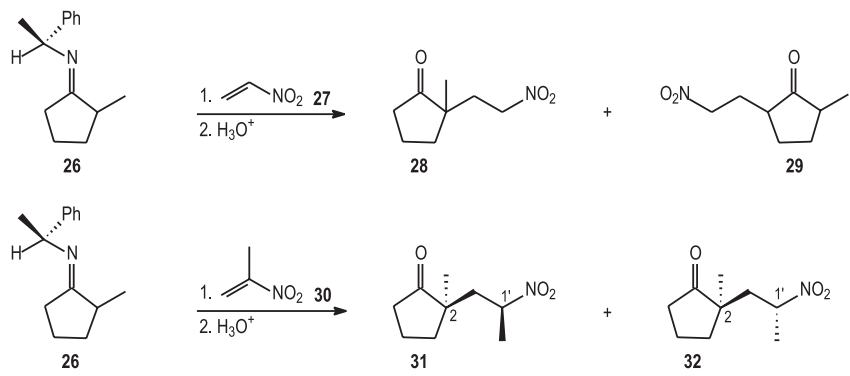

Esquema 6. Adição de Michael da imina (S)-26 ao nitroeteno (27) e ao 2nitropropeno (30)

Os autores não esclarecem estes resultados, porém verifica-se que a perda da regiosseletividade nestas reações ocorre devido à utilização de eletrófilos muito reativos, como 24 e 27.

Há na literatura, um trabalho referente à alquilação via imina quiral, da (3R)-2,3-dimetilcicloexanona (33), empregando o aceptor acrilato de metila (9), que apresenta um curso regioquímico diferente do normalmente observado para este aceptor ${ }^{22}$. Neste trabalho, a imina derivada da $(S)$-PEA, reage com o acrilato de metila (9) produzindo apenas o cetoéster 34, com 59\% de rendimento (Esquema 7). No entanto, a adição da imina derivada da $(R)$-PEA (3) ao acrilato de metila conduz aos adutos $35(0,3 \%), \mathbf{3 6}(1,5 \%), \mathbf{3 7}(0,4 \%), \mathbf{3 8}(0,3 \%)$ e $\mathbf{3 4}(2 \%)$, com baixa conversão e perda de regio- e estereosseletividade. Tais compostos foram somente separados através de HPLC ${ }^{22}$.

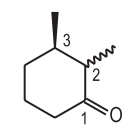

33

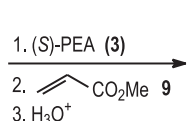

3. $\mathrm{H}_{3} \mathrm{O}$<smiles>CC(=O)CC[C@]1(C)C(=O)CCC[C@@H]1C</smiles>

34 $\mid \begin{aligned} & \text { 1. }(R)-\mathrm{PEA}(3) \\ & \text { 2. } \mathrm{CO}_{2} \mathrm{Me} 9\end{aligned}$<smiles>CC(=O)CC[C@H]1CC[C@@H](C)[C@H](C)C1=O</smiles><smiles>CC(=O)CC[C@H]1CC[C@@H](C)[C@@H](C)C1=O</smiles><smiles>CC(=O)CCC1CC[C@@H](C)[C@@H](C)C1=O</smiles><smiles>CC(=O)CC[C@H]1C(=O)CCC[C@@H]1C</smiles>

$38(2 \%)$

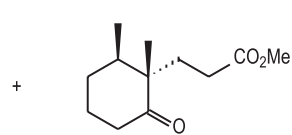

$34(0,3 \%)$

Esquema 7. Alquilação da (3R)-2,3-dimetilcicloexanona (33) empregando $o$ aceptor de Michael acrilato de metila (9) $e$ os auxiliares quirais $(S)-e$ (R)-PEA (3)

Segundo o autor, a adição da imina derivada da cetona $\mathbf{3 3}$ e da (S)-PEA (3) ao acrilato de metila (9) corresponderia a uma situação "matched" de dupla indução assimétrica ${ }^{23}$, onde há a formação de um único aduto de Michael - o cetoéster 34. Entretanto, a adição da imina derivada da cetona $\mathbf{3 3}$ e da $(R)$-PEA (3) ao acrilato resultaria

num caso "mismatched", onde o estado de transição não favoreceria a reação desejada. Este fenômeno foi interpretado pelo autor como sendo causado por interações não ligantes, no estado de transição, entre as espécies acrilato de metila, feniletilamina quiral e substituinte Me-3 do anel cicloexano ${ }^{22}$, que são mais claramente entendidas analisando-se o Modelo de Houk (ver adiante).

Resultados recentes, obtidos por nosso grupo de pesquisa ${ }^{24}$, relacionados à reação de alquilação desracemizante da (3R)-2,3dimetilcicloexanona (33) demonstraram que, na presença do auxiliar quiral $(S)$-PEA (3) e do aceptor metilvinilcetona (20), se produzia a mistura dos adutos quaternário (39) e terciários (40), na proporção de $3,4: 1$ e com $69 \%$ de rendimento, enquanto que, na presença do enantiômero $(R)$-PEA (3), se produzia, somente, a mistura diastereoisomérica dos adutos terciários $\mathbf{4 0}$, com $50 \%$ de rendimento (Esquema 8).

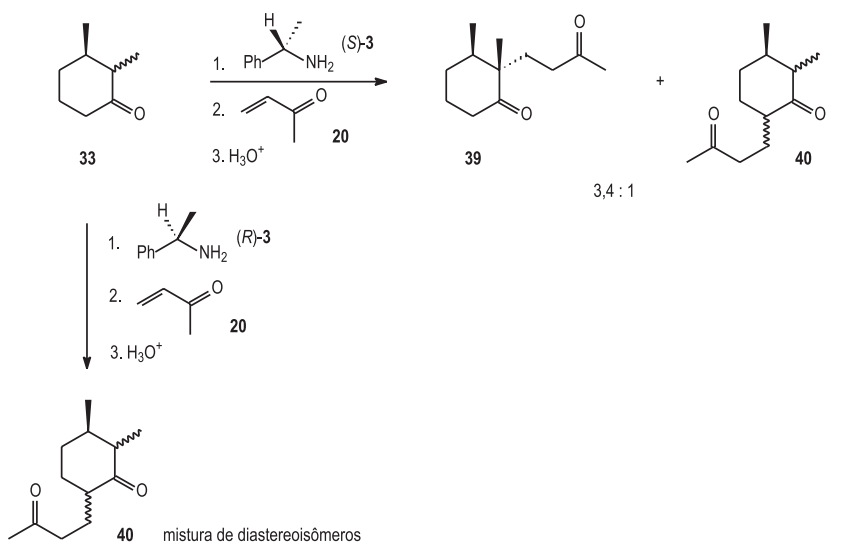

Esquema 8. Reação de alquilação da (3R)-2,3-dimetilcicloexanona (33), empregando o aceptor metilvinilcetona (20) e os auxiliares quirais (S)- $e$ (R)-PEA (3)

Estes resultados, referentes à adição de Michael assimétrica da imina derivada da (3R)-2,3-dimetilcicloexanona (33) aos aceptores metilvinilcetona (20) ou acrilato de metila (9) (eletrófilos que normalmente conduzem à regio- e estereosseletividade altas), indicam que a presença de um centro estereogênico pré-existente na enamina de partida conduz a uma perda de regiosseletividade, devido a efeitos de ordem conformacional no ciclo e a fatores de ordem estereoeletrônica no estado de transição que não estavam previstos nos modelos clássicos desta reação ${ }^{2-4}$.

\section{Considerações estereoquímicas}

A estereoquímica da adição de iminas quirais a alquenos eletrofílicos, segundo d'Angelo, está de acordo com o ataque do confôrmero mais estável do tautômero enamina secundária, pela face $\pi$ oposta ao substituinte fenila. O estado de transição desta reação é cíclico e a aproximação entre as espécies reagentes é endo-sin, permitindo a transferência interna e concertada de hidrogênio ${ }^{2-4,16}$.

Por exemplo, a adição da imina 11, derivada da $(R)$-PEA (3), ao cianeto de crotonila (41) produz a mistura das lactamas bicíclicas $\mathbf{4 2}$ e 43, cujos substituintes Me-4 e Me-4a estão em estereoquímica cis (Esquema 9) ${ }^{25}$.

Este resultado sugere que a reação procede via estado de transição cíclico, tipo cadeira, representado por 44, com a aproximação endo e arranjo "gauche" (sinclinal) entre as espécies reagentes, de acordo com a projeção de Newman 45 (Figura 2) ${ }^{25}$.

A transferência interna de próton, que acompanha a reação de 

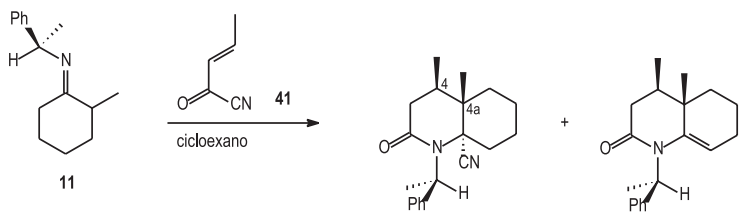

42

43

Esquema 9. Adição de Michael da imina 11 ao cianeto de crotonila (41)

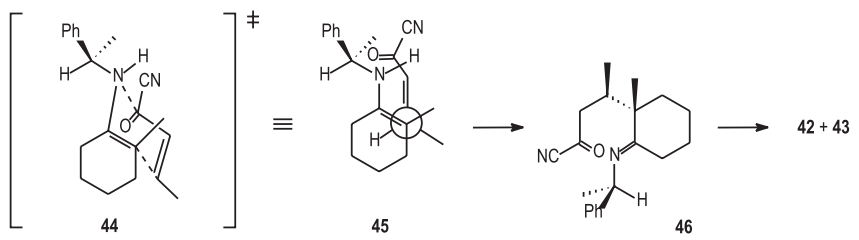

Figura 2. Estereoquímica da adição de Michael da imina 11 ao cianeto de crotonila (41)

alquilação desracemizante, foi estabelecida a partir dos resultados de De Jeso e Pommier ${ }^{26}$, onde a adição de enaminas deuteradas do tipo 47 a alquenos eletrofílicos produz os adutos $\mathbf{4 8}$, cujo átomo de deutério encontra-se somente em posição $\alpha$-GRE (Esquema 10). Esta tendência foi confirmada através de resultados obtidos com reagentes deuterados, conforme descrito anteriormente (Esquema 2) ${ }^{15}$.<smiles>[R]/C(C)=C/N([R])[R]</smiles>

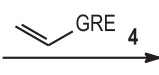

47

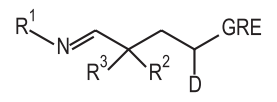

48
Esquema 10. Adição de uma enamina deuterada, tipo 47 a um alqueno eletrofílico, como 4

Resultados anteriores, obtidos por nosso grupo de pesquisa, relacionados à alquilação da $(R)$-diidrocarvona (49), evidenciaram que um centro estereogênico residual, presente no ciclo da enamina, é capaz de modificar radicalmente o curso estereoquímico desta reação ${ }^{27}$.

A adição de Michael das iminas quirais $\mathbf{5 0}$ e 53, ambas obtidas com rendimento de $89 \%$ a partir da $(R)$-diidrocarvona (49) e dos auxiliares $(S)$ - e $(R)$-PEA (3), à metilvinilcetona (20) conduziu ao primeiro caso observado de dupla indução assimétrica para a reação de alquilação via iminas quirais ${ }^{27}$. Numa situação "matched", a enamina $\mathbf{5 1}$ conduz, após alquilação, hidrólise e aldolização (NaOEt/ EtOH) do aduto de Michael, à octalona trans-dissubstituída 52, com e.d. $>95 \%$ e rendimento de $72 \%$. Entretanto, de acordo com a situação "mismatched", a enamina $\mathbf{5 4}$ produz, após alquilação, hidrólise e cuidadosa aldolização $\left(\mathrm{KOH} / \mathrm{EtOH}, 0{ }^{\circ} \mathrm{C}\right)$ do respectivo aduto de Michael, a mistura octalona cis 55 (52\%) e $\beta$-hidróxicetona 56 (14\%), facilmente separáveis por cromatografia (Esquema 11).

Tal perda de estereosseletividade, igualmente, deve ser causada por efeitos conformacionais e fatores de ordem estereoeletrônica no estado de transição, não previstos anteriormente.

Este caso "mismatched" permitiu a obtenção da octalona cisdissubstituída 55, de forma bastante eficiente se comparada com a literatura ${ }^{28-33}$. Como este intermediário sintético é de grande importância na preparação de sesquiterpenos eudesmanos, esta metodologia tem sido empregada por nosso grupo de pesquisa ${ }^{11}$, e também por outros grupos $^{13,14,34-38}$, na síntese de diversos produtos naturais com estas características estruturais.

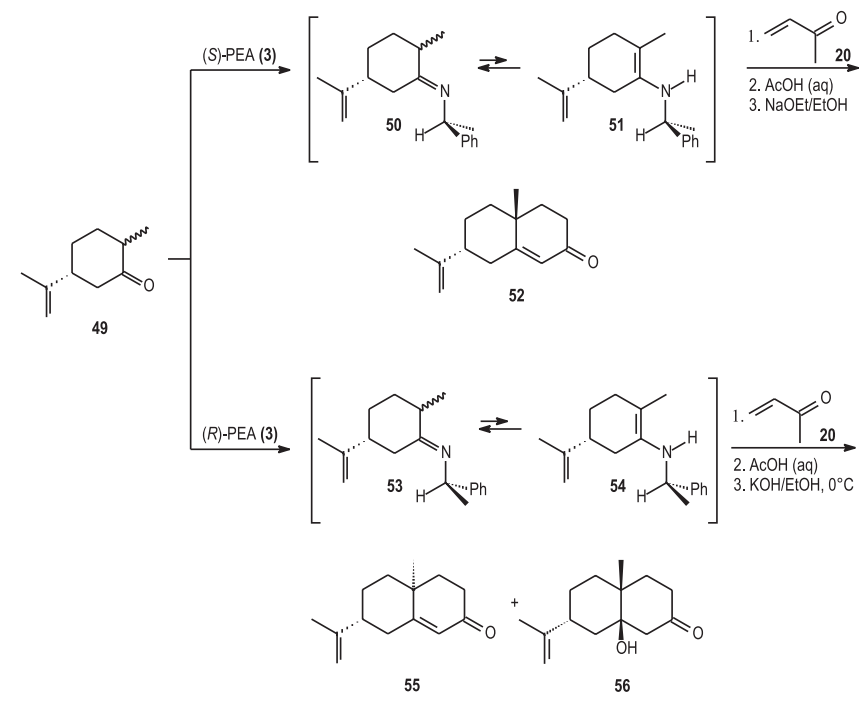

Esquema 11. Alquilação da (R)-diidrocarvona (49), empregando o aceptor metilvinilcetona (20) e os auxiliares quirais (S)- e (R)-PEA (3)

\section{Considerações sobre o estado de transição}

Cálculos $a b$ initio SCF-CI MO, referentes à adição de Michael da vinilamina ao propenal, evidenciaram que o estado de transição energeticamente favorecido é o de geometria cadeira 57 (Figura 3) ${ }^{16}$. Nesta geometria, há a estabilização extra do sistema devido a interações orbitalares entre o HOMO do doador (enamina) e o LUMO do aceptor (propenal). Ainda, os orbitais do nitrogênio e da carbonila interagem de forma ligante observando-se, inclusive, a atração secundária entre o oxigênio do propenal e C-1 da enamina, garantindo assim a aproximação endo entre as espécies reagentes (a energia da geometria cadeira é cerca de $4 \mathrm{kcal} / \mathrm{mol}$ menor que a energia da geometria barco 58) ${ }^{16}$. A transferência interna do hidrogênio da enamina para C-2 do propenal é facilitada, pois estes sítios estão próximos entre si. Esta transferência ocorre de forma mais ou menos concertada ao passo de adição, a fim de evitar, na medida do possível, o estado de transição zwitteriônico 60, que é energeticamente muito desfavorável. Portanto, a transferência concertada de hidrogênio não poderá ocorrer na geometria anti $\mathbf{5 9}$, ainda que esta apresente a menor energia, pois será induzida a formação do intermediário 60 (Figura 3). Resultados semelhantes estão descritos para a reação da vinilamina com formaldeído ${ }^{39}$.
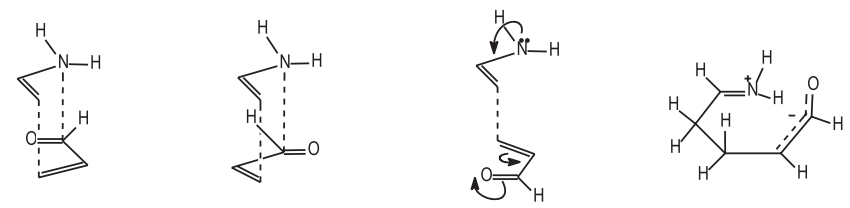

$57(8,79 \mathrm{kcal} / \mathrm{mol})$

$58(12,59 \mathrm{kcal} / \mathrm{mol})$

$59(7,81 \mathrm{kcal} / \mathrm{mol})$

$60(89,50 \mathrm{kcal} / \mathrm{mol})$

Figura 3. Energias, referentes às espécies vinilamina e propenal à distância infinita, nas principais geometrias no estado de transição

\section{Modelo de Houk para a indução assimétrica}

A indução assimétrica na reação de alquilação desracemizante, segundo Houk, está relacionada à transmissão conformacional da quiralidade (que se dá através do esqueleto do cicloalqueno) e não através do ataque anti ao grupo fenila ${ }^{40}$. Houk, através de cálculos $a b$ initio RHF (restrição Hartree-Fock) - 6-31G*, concluiu que as espécies $(N$-metilamino)etileno e acrilonitrila produzem um intermediá- 
rio zwitteriônico após a adição de Michael e, da mesma forma que Sevin ${ }^{16}$, via aproximação sin entre as espécies reagentes, devido à atração eletrostática entre os sítios carregados.

Ainda, a reação entre o ( $N$-metilamino)cicloexeno e a acrilonitrila apresenta dois estados de transição $\sin : \mathbf{6 1}$ e 62, cujos comprimentos de ligação C-C, em formação, são 1,872 e 1,846 ̊ - valores típicos para reações que produzem intermediários zwitteriônicos (Figura 4). Para a meia-cadeira do ( $N$-metilamino)cicloexeno, o ataque axial 61 é $2 \mathrm{kcal} / \mathrm{mol}$ mais estável que o ataque equatorial 62. A preferência pelo ataque axial em cicloexenos, enolatos e compostos similares é interpretada em função da tensão torcional. De acordo com a alquilação de enolatos e enóis, o ataque axial (estereoeletronicamente favorecido) passa por um estado de transição cadeira, ao passo que o ataque equatorial conduz a um complexo ativado tipo barco torci$\mathrm{do}^{40-42}$.

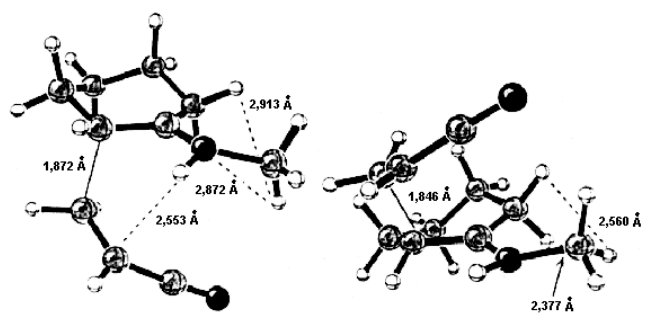

Figura 4. Estados de transição 61 (à esquerda) e 62 (à direita) - ataques axial e equatorial, na adição de Michael do (N-metilamino)cicloexeno à acrilonitrila. Reproduzido da ref. 40, com permissão da American Chemical Society

Para um aminocicloexeno aquiral, os dois confôrmeros meiacadeiras são enantioméricos e iguais em energia, de forma que o ataque axial, em cada um deles, é igualmente provável. Entretanto, na presença de um substituinte quiral, os dois confôrmeros são diastereoisoméricos e, conseqüentemente, um deles pode predomi$\operatorname{nar}^{40}$.

A análise conformacional dos enaminoésteres 63 e 64 não é realizada através de método $a b$ initio, devido ao número de átomos pertencente ao sistema. Assim, cálculos MM2 destas duas meia-cadeiras diastereoisoméricas demonstram que o confôrmero 63 é $0,8 \mathrm{kcal} /$ mol mais estável que o 64, devido a interações não ligantes menos efetivas no primeiro (Figura 5). Portanto, o ataque axial neste confôrmero é favorecido por um valor estimado de $1 \mathrm{kcal} / \mathrm{mol}$, em relação ao ataque na face oposta (resultados de barreira energética, obtidos através de mecânica molecular). Esta diferença de energia produz a razão estereoisomérica de 90:10, normalmente observada em reações de alquilação desracemizante. Ainda, a posição preferencial do grupo PEA não é aquela predita pelo modelo de Sevin ${ }^{16} \mathrm{e}$ sim, a que induz o hidrogênio do auxiliar quiral a se orientar, em direção ao anel cicloexeno, para produzir as distâncias H-H de acordo com a figura abaixo (Figura 5$)^{40}$.

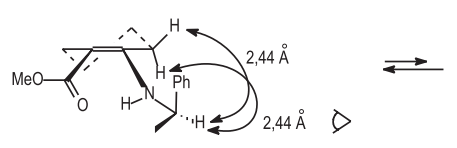

$63\left(\mathrm{E}_{\mathrm{rel}}=0,0 \mathrm{kcal} / \mathrm{mol}\right)$

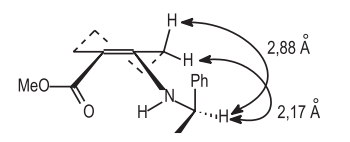

$64\left(\mathrm{E}_{\text {rel. }}=0,8 \mathrm{kcal} / \mathrm{mol}\right)$
Figura 5. Confôrmeros cadeira-torcidas 63 e 64

A transmissão conformacional da quiralidade fornece um modelo geral para a alta estereosseletividade obtida nas reações de alquilação desracemizante e, portanto, permite a explicação dos resultados regio- e estereoquímicos anômalos, como os apresentados anteriormente. O modelo de Houk leva em consideração os efeitos conformacionais no ciclo da enamina, evidentemente relevantes na presença de substituintes de anel.

Os confôrmeros 65 e 66, correspondentes à orientação da fenila em relação ao anel cicloexeno de uma enamina, possuem diferentes energias, sendo a primeira a mais estável termodinamicamente (Figura 6$)^{40}$.

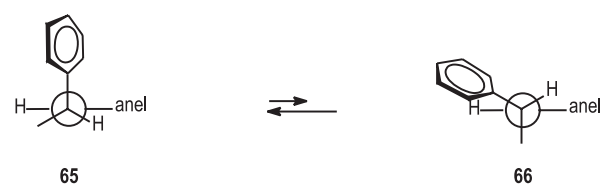

Figura 6. Confôrmeros 65 (majoritário) e 66 (minoritário)

De acordo com as estruturas acima, verifica-se o porquê da não variação do excesso enantiomérico quando se substitui a fenila ou a metila por grupos mais volumosos, como o naftaleno ou a isopropila ${ }^{2}$. Segundo Houk, as interações estéricas entre o substituinte aromático e o eletrófilo não correspondem à maior fonte de indução, considerando que o ataque axial, mais favorecido, se dá pela face oposta àquela onde se encontra o grupo fenila. Da mesma forma que, a substituição de metila por isopropila não afeta a seletividade da reação, porque não há alteração na conformação preferencial 65. O substituinte fenila permanece perpendicular ao anel e a diferença de energia entre os confôrmeros 65 e 66 continua sendo a mesma. Entretanto, observa-se uma queda acentuada na estereosseletividade da reação ( $45 \%$ e.e.), quando se substitui a fenila por cicloexila ou norbonila ${ }^{2}$. Neste caso, os confôrmeros 65 e 66 apresentariam energias quase idênticas ${ }^{40}$.

Um aspecto importante nos resultados de Houk ${ }^{40}$, apresentados nas figuras 5 e 6, é que os cálculos de mecânica molecular são realizados com enaminoésteres e não com enaminas. A extensão da conjugação em enaminoésteres conduz à hibridização $\mathrm{sp}^{2}$ do átomo de nitrogênio, simplificando os cálculos. Entretanto, esta não é a real situação quando se emprega enaminas, pois a geometria do átomo de nitrogênio é piramidal, comprovada através de dados experimentais ${ }^{18}$, tornando os cálculos muito mais complexos ${ }^{17}$. Portanto, ainda que o modelo de Houk corresponda a uma generalização consistente na descrição da reação de Michael com enaminas substituídas, na medida em que é o único modelo a discutir aspectos de ordem conformacional e, portanto, preferências estereoeletrônicas de ataque, ele não é capaz de fornecer uma descrição mais precisa da conformação ao redor da ligação $\mathrm{N}-\mathrm{C}^{*}$, visto que desconsidera a hibridização $\mathrm{sp}^{3}$ do átomo de nitrogênio.

\section{Modelo de Tran Huu Dau e d'Angelo para a indução assimétrica}

Há outro estudo teórico, realizado por Tran Huu Dau e d'Angelo, que inclui cálculos de modelagem molecular relacionados à discriminação $\pi$-facial de enaminocetonas quirais, em relação à adição de acrilato de metila ${ }^{43}$. Neste trabalho, o anel cicloexeno da enamina, conformacionalmente flexível, foi substituído pelo anel ciclopenteno que é planar. Conseqüentemente, a discriminação $\pi$-facial da enamina é governada apenas pelo substituinte quiral da espécie enaminocetona. Sabe-se que enaminas apresentam geometria tetraédrica para o átomo de nitrogênio ${ }^{18}$, o que dificulta os cálculos de modelagem molecular ${ }^{17}$. No entanto, em enaminocetonas o nitrogênio assume geometria planar, devido ao efeito de conjugação com o grupo carbonila, reduzindo um grau de liberdade da molécula. 
As estruturas dos estados de transição, geradas pela adição do acrilato de metila à enaminocetona $\mathbf{6 7}$, foram investigadas através de cálculos semi-empíricos AM1 (MOPAC-RHF). Os resultados para as duas estruturas diastereoisoméricas (aproximações às faces $R e \mathrm{e}$ Si de C-1), a $3 \AA$, evidenciaram que, quando o acrilato de metila se aproxima pela face $R e$ de C-1 (levando à formação do diastereoisômero majoritário), o rotâmero de menor energia da enaminocetona apresenta um ângulo diedro (C2-N-C7-C9) $\theta=60^{\circ} \mathrm{C}$. Entretanto, se o acrilato de metila se aproxima pela face $\mathrm{Si}$ de C-1 (levando à formação do diastereoisômero minoritário), o mínimo de energia corresponde a $\theta=180^{\circ}$, de forma que, para minimizar as interações estéricas, a fenila é empurrada para fora da sua posição mais estável (Figura 7$)^{43}$.
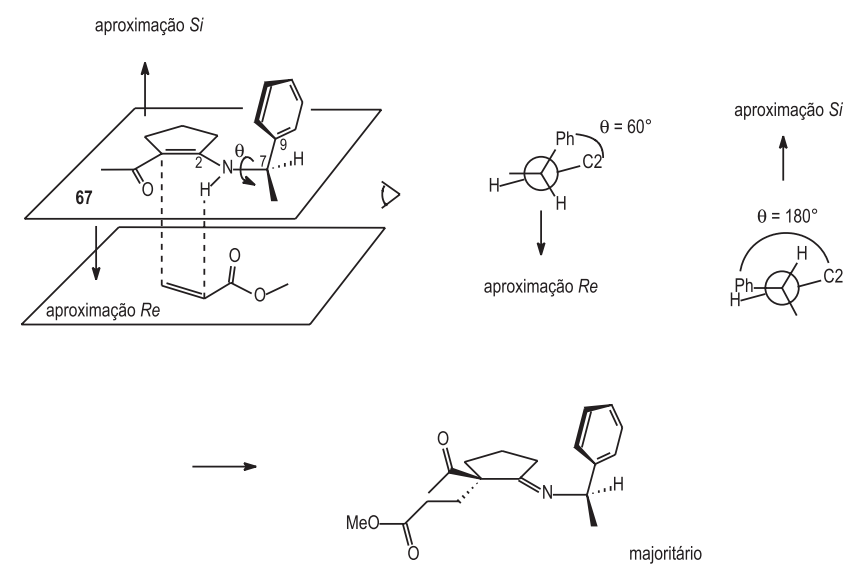

Figura 7. Aproximações Re e Si entre o acrilato de metila e a enaminocetona 67

Portanto, entre as duas estruturas diastereoisoméricas, à distância de $3 \AA$, a preferência conformacional produz a diferença de energia de $-0,9 \mathrm{kcal} / \mathrm{mol}$, na medida em que o ângulo diedro $\theta=60^{\circ}$ se assemelha muito ao obtido na forma cristalina $\left(\theta=81^{\circ}\right)^{44}$. As entalpias de formação das estruturas no estado de transição e a energia de ativação da reação correspondente estão resumidas na Tabela 1. A partir de cálculos AM1, as diferenças em energia, para 67, entre as duas estruturas de transição propostas, $\Delta \mathrm{H}_{R e}^{\ddagger}-\Delta \mathrm{H}_{S \mathrm{i}}^{\ddagger}=-2,2 \mathrm{kcal} /$ mol, concordam com os resultados experimentais de $95 \%$ de e.d..

A repulsão entre $\mathbf{6 7}$ e o acrilato de metila, na face $\mathrm{Si}$, é mais intensa que na face $R e\left(\Delta \mathrm{H}_{S i}-\Delta \mathrm{H}_{R e}=0,9 \mathrm{kcal} / \mathrm{mol}\right)$, devido a interações não ligantes. A otimização, por AM1, dos estados de transição para as aproximações $R e$ e $S i$ indica que fatores estéricos governam a geometria destas estruturas de transição (Figura 8). As interações relacionadas ao grupo PEA, nas aproximações $R e$ e $\mathrm{Si}$, estão caracterizadas por linhas pontilhadas ${ }^{43}$.

Portanto, na estrutura do estado de transição para a aproximação Si em 67, um átomo de hidrogênio da fenila do auxiliar quiral está próximo de um átomo de hidrogênio do substituinte metila do acrilato (2,19 A) e um átomo de hidrogênio da metila do auxiliar quiral está próximo do átomo de hidrogênio do anel de cinco membros
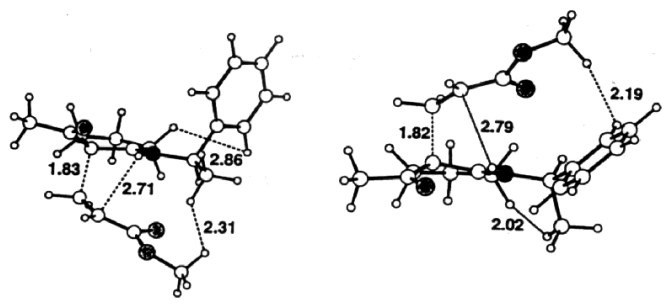

Figura 8. Estruturas de transição, por AM 1, para a adição da enaminocetona 67 ao acrilato de metila: aproximação Re, à esquerda e aproximação Si, à direita. Reproduzido da ref. 43, com permissão da Elsevier Science

$(2,02 \AA)$. A rotação em torno do eixo N-C7 pode aliviar uma das interações mas, inevitavelmente, aumentará a outra. Entretanto, na estrutura do estado de transição que leva à aproximação $R e$ em $\mathbf{6 7}$, as interações do auxiliar quiral com o anel de cinco membros, assim como com o acrilato de metila, são menos desestabilizantes.

Ainda nestas estruturas propostas, o comprimento da ligação CC em formação é 1,83 Å e o átomo de hidrogênio ligado ao nitrogênio não está distante de C2' do acrilato (2,71 ̊̊). Estes resultados, segundo os autores, são consistentes com a etapa de transferência interna e rápida de próton sendo esta adição de Michael interpretada como um processo tipo aza-ene, com fatores estéricos ditando a estereosseletividade da reação ${ }^{43}$. Porém, as simplificações do modelo de Tran Huu Dau e d'Angelo, eliminando as influências conformacionais no ciclo, não permitem que este possa ser empregado para justificar os resultados observados em enaminas substituídas conformacionalmente móveis, além do que tal modelo não leva em consideração a piramidalização do átomo de nitrogênio de enaminas sem conjugação estentida.

\section{Considerações sobre a regiosseletividade observada na reação de alquilação da $(3 R)$-2,3-dimetilcicloexanona $(33)$, pelo modelo de Houk}

Considerando os resultados regioquímicos da reação de alquilação da (3R)-2,3-dimetilcicloexanona (33), obtidos por nosso grupo de pesquisa $^{24}$, e relacionando-os com o trabalho publicado por Houk ${ }^{40}$, propõe-se:

1 - para a reação de alquilação da (3R)-2,3-dimetilcicloexanona (33), empregando o auxiliar quiral $(S)$-PEA (3), há dois fatores que desestabilizam o confôrmero 68a, da enamina secundária mais substituída: i) a forte interação estérica entre os substituintes Me-2 e Me3 (em posição pseudo-equatorial) (ângulo diedro $\left.\sim 39^{\circ}\right)^{45}$, devido à tensão alílica $\mathrm{A}^{1,2}$ e ii) as distâncias $\mathrm{H}($ anel) - $\mathrm{H}$ (auxiliar quiral) que correspondem ao confôrmero meia-cadeira de maior energia, em analogia aos resultados apresentados por $\operatorname{Houk}^{40}$ (Esquema 12).

Entretanto, a meia-cadeira $\mathbf{6 8 b}$, cujo substituinte Me-3 encontra-se em pseudo-axial aliviando a interação $\mathrm{A}^{1,2}$, apresenta as distâncias H(anel) - H(auxiliar quiral) correspondentes ao confôrmero

Tabela 1. $\Delta \mathrm{H}_{\mathrm{f}}$ de 67 + acrilato de metila, a $3 \AA$ e das estruturas teóricas de transição, em kcal/mol. $\Delta \mathrm{E}=$ diferença entre a soma das entalpias das espécies reagentes no estado fundamental e das correspondentes estruturas de transição mais estáveis

\begin{tabular}{ccccccc}
\hline \multicolumn{3}{c}{ Espécies Reagentes a $3 \AA$} & \multicolumn{3}{c}{ AM1 - Energias de Ativação } \\
\hline$\Delta \mathrm{H}_{\mathrm{Re}}$ & $\Delta \mathrm{H}_{\mathrm{Si}}$ & $\Delta \mathrm{H}_{\mathrm{Re}}-\Delta \mathrm{H}_{\mathrm{Si}}$ & $\Delta \mathrm{H}_{\mathrm{Re}}^{\ddagger}$ & $\Delta \mathrm{H}_{\mathrm{Si}}^{\ddagger}$ & $\Delta \mathrm{H}_{\mathrm{Re}}^{\ddagger}-\Delta \mathrm{H}_{\mathrm{Si}}^{\ddagger}$ & $\Delta \mathrm{E}$ \\
$-85,2$ & $-84,3$ & $-0,9$ & $-61,9$ & $-59,7$ & $-2,2$ & $-27,3$ \\
\hline
\end{tabular}




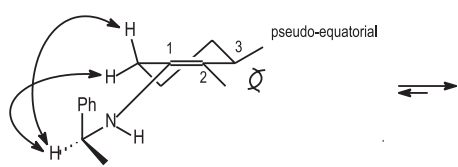

68 a

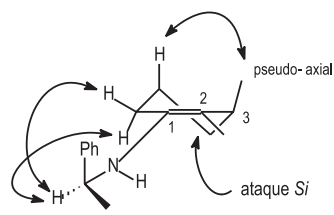

$68 \mathrm{~b}$
Esquema 12. Confôrmeros $\boldsymbol{a}$ e $\boldsymbol{b}$ da enamina 68, derivada da (S)-PEA (3)

de menor energia, de acordo com Houk. Esta junção de fatores determina a maior estabilidade do confôrmero $68 \mathbf{b}$ em relação ao $68 \mathbf{a}$. A adição de Michael ocorrerá por ataque axial (estericamente e estereoeletronicamente favorecidos) à face $S i$ do confôrmero mais estável da enamina 68 (Figura 9). Esta situação conduz ao caso "matched" de dupla indução assimétrica ${ }^{23}$, onde há a formação de um único diastereoisômero do aduto quaternário - a dicetona cis 39.

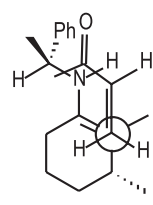

68

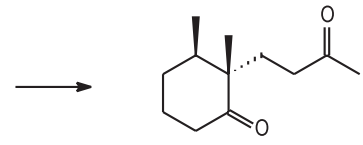

39
Figura 9. Aproximação da metilvinilcetona à face Si da enamina $\mathbf{6 8}$

No entanto, a perda parcial da regiosseletividade, observada nesta reação, e a conseqüente formação das dicetonas regioisoméricas $\mathbf{4 0}$, pode ser explicada em função das interações 1,3-diaxiais entre os substituintes Me-3 e H-5, no confôrmero $\mathbf{6 8 b}$, que o desestabiliza induzindo a adição de Michael via a enamina secundária menos substituída 69 (Figura 10).

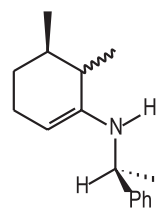

Figura 10. Tautômero enamina secundária menos substituída 69

2 - Para a reação de alquilação da (3R)-2,3-dimetilcicloexanona (33), empregando o auxiliar quiral $(R)$-PEA (3), observam-se as seguintes interações na enamina mais substituída 70 (Esquema 13): i) o confôrmero 70a não é estabilizado, devido à tensão por interação alílica entre os substituintes Me-2 e Me-3 (em posição pseudo-equatorial), embora as distâncias $\mathrm{H}($ anel) $-\mathrm{H}$ (auxiliar quiral) conduzam à meia-cadeira de maior estabilidade, segundo Houk e ii) a conformação 70b, cuja Me-3 encontra-se em pseudo-axial, não apresenta interação $\mathrm{A}^{1,2}$, mas sim interação 1,3-diaxial (entre Me-3 e H-5) e, ainda, as distâncias $\mathrm{H}($ anel $)$ - H(auxiliar quiral) correspondem à situação de maior energia, de acordo com Houk (Esquema 13). Portanto, nenhum dos confôrmeros a ou b, da enamina $\mathbf{7 0}$, é favorecido

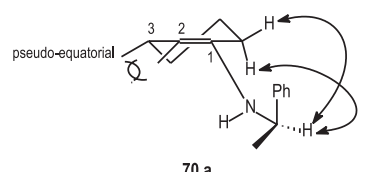

70 a

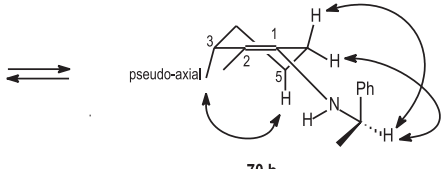

$70 \mathrm{~b}$
Esquema 13. Confôrmeros $\boldsymbol{a}$ e $\boldsymbol{b}$ da enamina 70, derivada da (R)-PEA (3) no equilíbrio e a adição de Michael dá-se exclusivamente via a enamina secundária menos substituída 71 (Esquema 14).

A adição de Michael das enaminas secundárias menos substituídas 69 e 71 à metilvinilcetona (20) pode ser racionalizada considerando os dois modelos de indução assimétrica:

i) Modelo de Sevin: para que haja a transferência interna e concertada de hidrogênio, a geometria da ligação N-H deve ser sin à ligação dupla da enamina, de forma que a barreira energética, da conversão entre os rotâmeros a e b, da enamina $\mathbf{7 1}$ (derivada da cetona 33 e da (R)-PEA (3)), deve ser ultrapassada (Esquema $14)$.<smiles>CC1CCC=C(NC(C)(C)C)C1C</smiles>

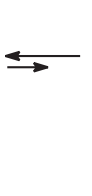<smiles>CC1CCC=C2NC(C)CC2C1</smiles>

$71 \mathrm{~b}$

Esquema 14. Rotâmeros $\boldsymbol{a}$ e $\boldsymbol{b}$ da enamina menos substituída 71

ii) Modelo de Houk: os rotâmeros mais estáveis das enaminas menos substituídas, representados por 69 e 71a, cujas ligações N-H estão anti à ligação dupla do anel, não conduzem à transferência concertada de hidrogênio.

Considerações sobre a diastereosseletividade observada na reação de alquilação da $(R)$-diidrocarvona $(47)$, pelo modelo de Houk

A estereoquímica da reação de alquilação da $(R)$-diidrocarvona $(49)^{27}$, empregando a $(S)$-PEA (3), pode ser avaliada considerando os confôrmeros a e b da enamina secundária 51. Observa-se que 51a é mais estável, pois o grupo isopropenila encontra-se em equatorial e as distâncias $\mathrm{H}($ anel $)$ - H(auxiliar quiral) correspondem à meiacadeira de maior estabilidade, de acordo com Houk (Esquema 15). $\mathrm{O}$ ataque axial (estericamente e estereoeletronicamente favorecidos) no confôrmero mais estável, 51a, conduz à situação "matched" de indução assimétrica dupla ${ }^{23}$, onde o substrato e o auxiliar quiral trabalham para a formação de um único produto - a dicetona $\mathbf{7 2}$.
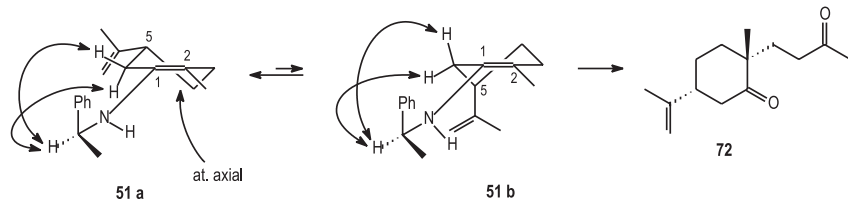

$51 \mathrm{~b}$

Esquema 15. Confôrmeros $\boldsymbol{a}$ e $\boldsymbol{b}$ da enamina 51, derivada da (S)-PEA (3)

Para a reação de alquilação de 49, empregando a (R)-PEA (3), observam-se as seguintes interações na enamina secundária mais substituída 54: i) o confôrmero a apresenta as distâncias H(anel) $\mathrm{H}$ (auxiliar quiral) relativas à meia-cadeira de maior estabilidade, segundo Houk, porém o substituinte isopropenila, em axial, sofre interação 1,3-diaxial com H-3 e ii) o confôrmero b apresenta as distâncias $\mathrm{H}$ (anel) - $\mathrm{H}$ (auxiliar quiral) referentes à meia-cadeira de menor estabilidade, porém o grupo isopropenila, em equatorial, induz estabilidade a 54b (Esquema 16). Esta junção de fatores conduz à situação "mismatched" de indução assimétrica dupla, onde o substrato e o auxiliar quiral trabalham em direções opostas, induzindo à formação das dicetonas $\mathbf{7 2}$ e $\mathbf{7 3}$. 

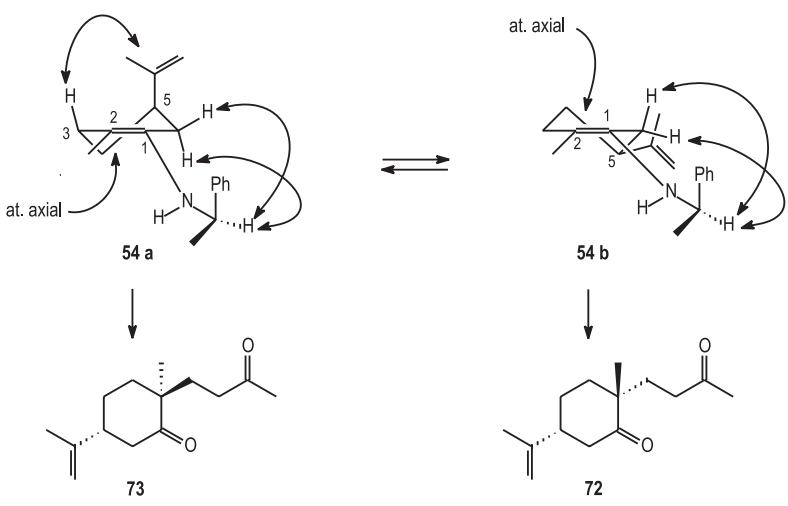

Esquema 16. Confôrmeros $\boldsymbol{a}$ e $\boldsymbol{b}$ da enamina 54, derivada da (R)-PEA (3)

A formação de $\mathbf{7 3}$ dá-se pela aproximação à face $R e$ da enamina 54, por ataque axial (estereoeletronicamente favorecido) no confôrmero a, enquanto 72 é obtido por ataque axial no confôrmero b.

Considerações sobre a diastereosseletividade observada na reação de alquilação da $(3 R, 4 R)-(-)-4-t$-butildimetilsiloxi)-2,3dimetilcicloexanona (74), pelo modelo de Houk

Há na literatura um trabalho, relacionado à síntese dos sesquiterpenos eremofilanos (+)-petasina e (+)-isopetasina, que apresenta os resultados da reação de alquilação desracemizante da $(3 R, 4 R)$-(-)-4-( $t$-butildimetilsiloxi)-2,3-dimetilcicloexanona (74), empregando o auxiliar ( $R$ )-PEA (3) e o aceptor acrilato de metila (9). Neste caso de alquilação de uma enamina com centros estereogênicos pré-existentes, o autor indica uma seletividade, não especificada (que deve ser regiosseletividade), de 8:1 na formação do aduto de Michael 75 (Esquema 17) ${ }^{46}$. Este aduto é obtido, em proporção superior a 95:5, como mistura de diastereoisômeros.

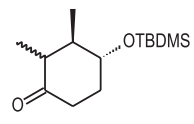

74

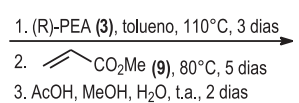

$(77 \%)$

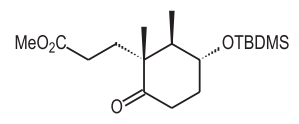

75
Esquema 17. Alquilação da (3R,4R)-(-)-4-(t-butildimetilsiloxi)-2,3dimetilcicloexanona (74), empregando o auxiliar (R)-PEA (3) e o aceptor acrilato de metila (9)

Embora o autor não tenha justificado a seletividade de 8:1 encontrada, os resultados obtidos podem ser racionalizados de acordo com o modelo de Houk, que leva em consideração efeitos conformacionais no ciclo de enaminas substituídas. O confôrmero 76a é mais favorecido que 76b, devido às distâncias H(anel) $\mathrm{H}$ (auxiliar quiral), que conduzem à meia-cadeira de maior estabilidade. Ainda, 76b apresenta tensão alílica $\mathrm{A}^{1,2}$, entre os substituintes metila em C-2 e C-3, que é mais desestabilizante que as interações 1,3-diaxiais entre os grupos alquilsililóxi, em C-4 e metila, em C-3, com os hidrogênios em C-5 e C-6 de 76a. Portanto, o ataque axial à face $R e$ em 76a produziria a diastereosseletividade observada (e.d. $>95 \%$ ) (Esquema 18), ainda que este confôrmero apresente os grupos metila em C-3 (pseudo axial) e alquilsililóxi em C-4 (axial), induzindo sua desestabilização. Entretanto, o ataque axial no confôrmero 76b produziria o epímero em C-2 de 75, não encontrado pelo autor, o que deve ser indicativo da alta energia deste confôrmero em relação a $\mathbf{7 6 a}$.

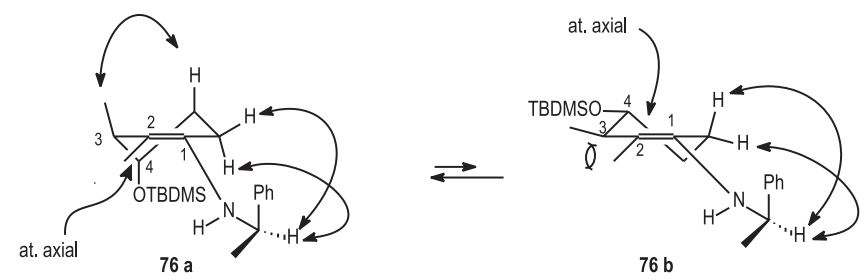

Esquema 18. Análise conformacional de $76 a$ e $76 b$

Ainda, as fortes interações estéricas, em ambos confôrmeros, podem ter conduzido à adição de Michael via enamina menos substituída, produzindo a regioseletividade observada de 8:1, ainda que não especificada pelo autor.

\section{CONSIDERAÇÕES FINAIS}

A adição de Michael, via iminas quirais derivadas da 1feniletilamina, constitui um dos métodos mais eficientes na elaboração estereocontrolada de centros carbônicos quaternários. Esta reação é, em geral, bastante regio- e estereosseletiva.

Neste trabalho discutiu-se a forte influência de centros estereogênicos pré-existentes, no ciclo da enamina, sobre a regio- e a estereosseletividade da reação de alquilação desracemizante. Efeitos de ordem conformacional no ciclo, induzidos por estes centros, não podem ser negligenciados e fatores de ordem estereoeletrônica devem ser considerados no estado de transição.

Tais fatores são menosprezados nos modelos convencionais (Sevin, d'Angelo e Tran Huu Dau) para esta reação. No entanto, o modelo proposto por Houk, que privilegia a transmissão conformacional da quiralidade, preconiza a diastereosseleção facial como função dos fatores estereoeletrônicos, normalmente presentes no ciclo da enamina secundária. Este modelo foi empregado com sucesso por nosso grupo, justificando as baixas regio- e estereosseletividades obtidas na adição de Michael das enaminas derivadas da (3R)-2,3dimetilcicloexanona e $(7 R)$-diidrocarvona, ambas oriundas da $(R)$ 1-feniletilamina, à metilvinilcetona.

Portanto, o controle da regio- e da estereosseletividade desta reação se dá através do ataque estereoeletronicamente favorecido ao confôrmero mais estável da enamina secundária (determinado pelos substituintes presentes no ciclo). Em geral, obtém-se alto grau de regio- e estereosseletividade, porém quando os dois centros estereogênicos (o pré-existente na enamina e o do auxiliar quiral) não promovem uma interação construtiva (situação "mismatched") as seletividades caem, pois várias conformações da enamina podem coexistir em equilíbrio.

\section{AGRADECIMENTOS}

Ao CNPq, à FAPERGS e à PUCRS, pelo suporte financeiro.

\section{REFERÊNCIAS}

1. Pfau, M.; Revial, G.; Guingant, A.; d'Angelo, J.; J. Am. Chem. Soc. 1985 , 107, 273.

2. d'Angelo, J.; Desmaële, D.; Dumas, F.; Guingant, A.; Tetrahedron: Asymmetry 1992, 3, 459 .

3. d'Angelo, J.; Cavé, C.; Desmaële, D.; Dumas, F. Em Trends in Organic Chemistry; Pandalai, S. G., ed.; Trirandrum: India, 1993, vol. 4, p. 555615

4. d'Angelo, J.; Cavé, C.; Desmaële, D.; Gassama, A.; Thominiaux, C.; Riche, C.; Heterocycles 1998, 47, 725.

5. Goes, A. J.; Cavé, C.; d'Angelo, J.; Tetrahedron Lett. 1998, 39, 1339.

6. Nour, M.; Tan, K.; Cavé, C.; Villeneuve, D.; Desmaële, D.; d'Angelo, J.; Riche, C.; Tetrahedron: Asymmetry 2000, 11, 995. 
7. Keller, L.; Camara, C.; Pinheiro, A.; Dumas, F.; d'Angelo, J.; Tetrahedron Lett. 2001, 42, 381.

8. Keller, L.; Dumas, F.; d'Angelo, J.; Tetrahedron Lett. 2001, 42, 1911.

9. Thominaux, C.; Roussé, S.; Desmaële, D.; D’Angelo, J.; Riche, C.; Tetrahedron: Asymmetry 1999, 10, 2015.

10. d'Angelo, J.; Revial, G.; Costa, P. R. R.; Castro, R. N.; Antunes, O. A. C.; Tetrahedron: Asymmetry 1991, 2, 199.

11. Tenius, B. S. M.; Rohde, A. R.; Victor, M. M.; Viegas Jr., C.; Synth. Commun. 1996, 26, 197.

12. Alves, J. C. F.; Simas, A. B. C.; Costa, P. R. R.; Tetrahedron: Asymmetry 1999, 10, 297.

13. Zhabinskii, V. N.; Minaard, A. J.; Wijnberg, J. B. P. A.; de Groot, A.; J. Org. Chem. 1996, 61, 4022.

14. Xiong, Z. M.; Zhou, G.; Yang, J.; Chen, Y. G.; Li, Y. L.; Tetrahedron: Asymmetry 1998, 9, 1525.

15. Ambroise, L; Desmaële, D.; Mahuteau, J.; d'Angelo, J.; Tetrahedron Lett. 1994, 35, 9705 .

16. Sevin, A.; Tortajada, J.; Pfau, M. J.; Org. Chem. 1986, 51, 2671.

17. Sevin, A.; Masure, D.; Giessner-Prettre, C.; Pfau, M.; Helv. Chim. Acta 1990, 73, 552 .

18. Brown, K. L.; Damm, L.; Dunitz; J. D.; Eschenmoser, A.; Hobi, R.; Kratky, R.; Helv. Chim. Acta 1978, 61, 3108.

19. Jabin, I.; Revial, G.; Tomas, A.; Lemoine, P.; Pfau, M.; Tetrahedron: Asymmetry 1995, 6, 1795.

20. Volpe, T.; Revial, G.; Pfau, M.; d'Angelo, J.; Tetrahedron Lett. 1987, 28, 2367.

21. Pinheiro, S.; Desmaële, D.; Guingant, A.; d'Angelo, J.; Tetrahedron: Asymmetry 1992, 3, 1003.

22. Tori, M.; Hisazumi, K.; Wada, T.; Sono, M.; Nakashima, K.; Tetrahedron: Asymmetry 1999, 10, 961.

23. Masamune, S.; Choy, W.; Petersen, J. S.; Sita, L. R.; Angew. Chem., Int. Ed. 1985, 24, 1.

24. Schenato, R. A.; dos Santos, E. M.; Tenius, B. S. M.; Costa, P. R. R.; Caracelli, I.; Zukerman-Schpector, J.; Tetrahedron: Asymmetry 2001, 12, 579 .
25. d'Angelo, J.; Guingant, A.; Riche, C.; Chiaroni, A.; Tetrahedron Lett. 1988, $29,2667$.

26. De Jeso, B.; Pommier, J. C.; J. Chem. Soc., Chem. Comm. 1977, 565.

27. Tenius, B. S. M.; de Oliveira, E. R.; Ferraz, H. M. C.; Tetrahedron: Asymmetry 1993, 4, 633

28. Marshall, J. A.; Fanta, W. I.; J. Org. Chem. 1964, 29, 2501

29. Humber, D. C.; Pinder, A. R.; Williams, R. A.; J. Org. Chem. 1967, 32, 2335.

30. Caine, D.; Gupton. III, J. T.; J. Org. Chem. 1974, 39, 2654.

31. Ziegler, F. E.; Hwang, K. J.; J. Org. Chem. 1983, 48, 3349.

32. Jansen, B. J. M.; Kreuger, J. A.; de Groot, A.; Tetrahedron 1989, 45, 1447.

33. Agami, C.; Kadouri-Puchot, C.; Le Guen, V.; Tetrahedron: Asymmetry 1993, 4, 641

34. Xiong, Z.; Yang, J.; Li, Y.; Tetrahedron: Asymmetry 1996, 7, 2607.

35. Chen, Y.; Xiong, Z.; Zhou, G.; Yang, J.; Li, Y.; Chem. Lett. 1997, 1289.

36. Xiong, Z.; Zhou, G.; Chen, Y.; Li, Y.; J. Chem. Res., Synop. 1998, 450.

37. Zhou, G.; Xiong, Z.; Chen, Y.; Li, Y.; J. Chem. Res., Synop. 1998, 650.

38. Chen, Y.; Xiong, Z.; Zhou, G.; Liu, L.; Li, Y.; Tetrahedron: Asymmetry 1998, 9, 1923.

39. Sevin, A.; Maddaluno, J.; Agami, C.; J. Org. Chem. 1987, 52, 5611.

40. Lucero, M.J.; Houk, K. N.; J. Am. Chem. Soc. 1997, 119, 826.

41. Eliel, E. L.; Wilen, S. H.; Stereochemistry of Organic Compounds, WileyInterscience: New York, 1994, p. 898.

42. Evans, D. A.; Asymmetric Synthesis, Morrison, J. D., ed.; Academic Press, New York, 1984, vol. 3, p. 26-28.

43. Tran-Huu-Dau, M. E.; Riche, C.; Dumas, F.; d'Angelo, J.; Tetrahedron: Asymmetry 1998, 9, 1059.

44. d'Angelo, J.; Revial, G.; Guingant, A.; Riche, C.; Chiaroni, A.; Tetrahedron Lett. 1989, 30, 2645.

45. Johnson, F.; Chem. Rev. 1968, 68, 375; ver também a correção em Chem. Rev. 1968, 68, 808.

46. Witschel, M.C.; Bestmann, H. J.; Synthesis 1997, 107. 